$\Delta$ Palabras clave/ Vivienda social, vivienda popular, ambiente térmico interior, clima cálido-húmedo.

$\Delta$ Keywords/ Social housing, popular housing, indoor thermal environment, warm-humid weather.

$\Delta$ Recepción/ 22 diciembre 2017

$\Delta$ Aceptación/ 27 abril 2018

\section{Vivienda de interés social en Portoviejo. Ambiente térmico interior.}

\author{
Social Housing in Portoviejo. \\ Indoor Thermal Environment.
}

\section{José Véliz-Párraga}

Arquitecto, Universidad Laica Eloy Alfaro de

Manabí, Ecuador.

Profesor, Universidad Técnica de Manabí, Ecuador.

jfveliz@hotmail.com

\section{Dania González-Couret}

Arquitecto, Universidad Tecnológica de La

Habana José Antonio Echeverría (CUJAE), Cuba. Doctor en Ciencias, Universidad Tecnológica de La Habana José Antonio Echeverría (CUJAE), Cuba.

Profesora, Universidad Tecnológica de La Habana José Antonio Echeverría (CUJAE), Cuba dania@arquitectura.cujae.edu.cu

RESUMEN/ La preocupación por la calidad de la vivienda social se ha incrementado, pero en su producción sigue predominando el interés por los costos más que por su habitabilidad. Esta investigación experimental exploratoria compara la influencia relativa de la tipología arquitectónica, la orientación y los materiales de construcción en el ambiente térmico interior de viviendas sociales y populares en sectores urbanos periféricos de la ciudad de Portoviejo, Ecuador. Para ello, se realizaron mediciones en 92 casos de estudio en las urbanizaciones donde el Ministerio de Desarrollo Urbano y Vivienda de Ecuador (MIDUVI) ha construido más viviendas. Los resultados obtenidos permiten verificar el efecto de la isla de calor urbana. La influencia del contexto y la tipología arquitectónica no resultan significativas en las urbanizaciones periféricas estudiadas. Las paredes de bambú coinciden con las mejores condiciones térmicas interiores y las cubiertas de hormigón con las peores. Existe correspondencia entre los resultados de la investigación experimental y la percepción de la población. ABSTRACT/ There is growing concern about the quality of social housing, but production still prioritizes costs over habitability. This exploratory and pilot research compares the relative influence of architectural typology, orientation and construction materials on the indoor thermal environments of social housing in the urban outskirts of the city of Portoviejo, Ecuador. Measurements were taken in 92 case studies, in urban complexes where Ecuador's Ministry of Housing and Urban Development (MIDUVI) has built more houses. Findings point to the urban heat island effect. The influence of the context and the architectural typology is not significant on the peripheral urban developments studied. While bamboo walls are consistent with the best indoor thermal conditions, concrete slabs are the worst. In addition, pilot research findings match the perceptions by the community.

\section{INTRODUCCIÓN.}

de 'vivir bien' en Bolivia o 'el buen vivir' Ecuador, inspiradas en la tradición indígena, buscan el equilibrio con la naturaleza en la satisfacción de las necesidades. Es así que en otros países, la preocupación por la calidad de la vivienda social se ha incrementado recientemente, como lo demuestran los casos México (Staines 2013; García, Bojórquez y Ruiz 2011; Romero 2010), Colombia (Ramos 2012), Chile (Espinosa y Cortés 2015) y Argentina (Vargas, Walsh y Barros 2016). Allí se ha puesto particular atención al ambiente interior, no solo por su importancia para la salud y el bienestar, sino, además, por el impacto ambiental generado como consecuencia del consumo de energía en iluminación, ventilación e, incluso, climatización artificial.

Sin embargo, la vivienda social promovida por el Estado en los países en desarrollo aún se caracteriza por soluciones arquitectónicas repetitivas, donde predomina el interés por minimizar los costos, más que una adecuada habitabilidad (Pérez 2013; Alonso, Sarachu y Vallés 2012; Cravino 2012), entendida esta como sus condiciones para ser habitada con satisfacción por parte los ocupantes y cuyos requerimientos a diferentes escalas varían según momento y lugar, de manera que quedan definidos en normas y regulaciones que deben ser sistemáticamente revisadas. En regiones cálido-húmedas, el ambiente térmico interior es afectado por el calor transmitido a través de la envolvente, como consecuencia de diseños y materiales inadecuados. Algunas prácticas y recomendaciones resultantes de investigaciones realizadas en países desarrollados situados en altas latitudes 
con climas fríos, pueden no ser apropiadas para climas cálido-húmedos en latitudes tan bajas como el Ecuador, por ejemplo, con respecto a la orientación. Por otro lado, algunas tendencias, aspiraciones y creencias predominantes, tanto en la población como en profesionales e instituciones, pueden generar en esas regiones impactos negativos que deben ser demostrados. Es por ello que la investigación experimental con carácter exploratorio cuyos resultados se presentan en este artículo, intenta comparar la influencia relativa de variables como la tipología arquitectónica, la orientación y los materiales de construcción en el ambiente térmico interior de viviendas de interés social y popular autoconstruidas en sectores urbanos periféricos de la ciudad de Portoviejo, Ecuador. Los resultados permiten caracterizar parcialmente el efecto de la isla de calor en los sectores urbanos estudiados.

\section{MARCO TEÓRICO. VARIABLES DEL OBJETO DE ESTUDIO. Durante la primera} etapa de la investigación se llevó a cabo una revisión de la literatura disponible sobre el tema, con la finalidad de identificar y caracterizar las variables arquitectónicas y urbanas que influyen en el ambiente térmico interior de las viviendas, a partir de los procesos globales que condicionan el hábitat urbano y los principios para lograr el cumplimiento del Objetivo 11 de los Objetivos de Desarrollo Sostenible (ODS)' Los resultados de la discusión teórica se resumen en la tabla 1, ya publicada por los autores (González y Véliz 2016)

Las variables coinciden con la clasificación de variables de diseño que condicionan el ambiente térmico interior propuesta por González (1997): contexto, volumen y orientación, espacio y envolvente. La morfología del contexto urbano influye en el microclima de los espacios exteriores, particularmente en la temperatura y el flujo del viento y, por lo tanto, en el ambiente térmico interior. La geometría urbana, mediante los parámetros que la definen y los que modifican su efecto, influye en la isla de calor urbana. La sección de las vías y la continuidad y regularidad de sus cierres, así como la compacidad y rugosidad de la masa edificada, caracterizan la morfología urbana, y su influencia en el microclima es modificada por la orientación, la topografía la vegetación y el albedo (González y Sánchez 2016).

Cuando se trata de contextos urbanos periféricos en proceso de ocupación, no existe una trama vial con secciones de vía en forma de cañón, delimitadas por las fachadas de las edificaciones que se alinean a ambos lados de la calle y que constituyen su cierre, sino que, por el contrario, se trata de un hábitat disperso con edificaciones de una o dos plantas y bajo albedo, por el predominio de vegetación en lugar de áreas pavimentadas

OBJETIVOS DE DESARROLLO SOSTENIBLE: Lograr que las ciudades y los asentamientos humanos sean inclusivos, seguros, resilientes y sostenibles

\section{PROCESOS Y ENFOQUES GLOBALES}

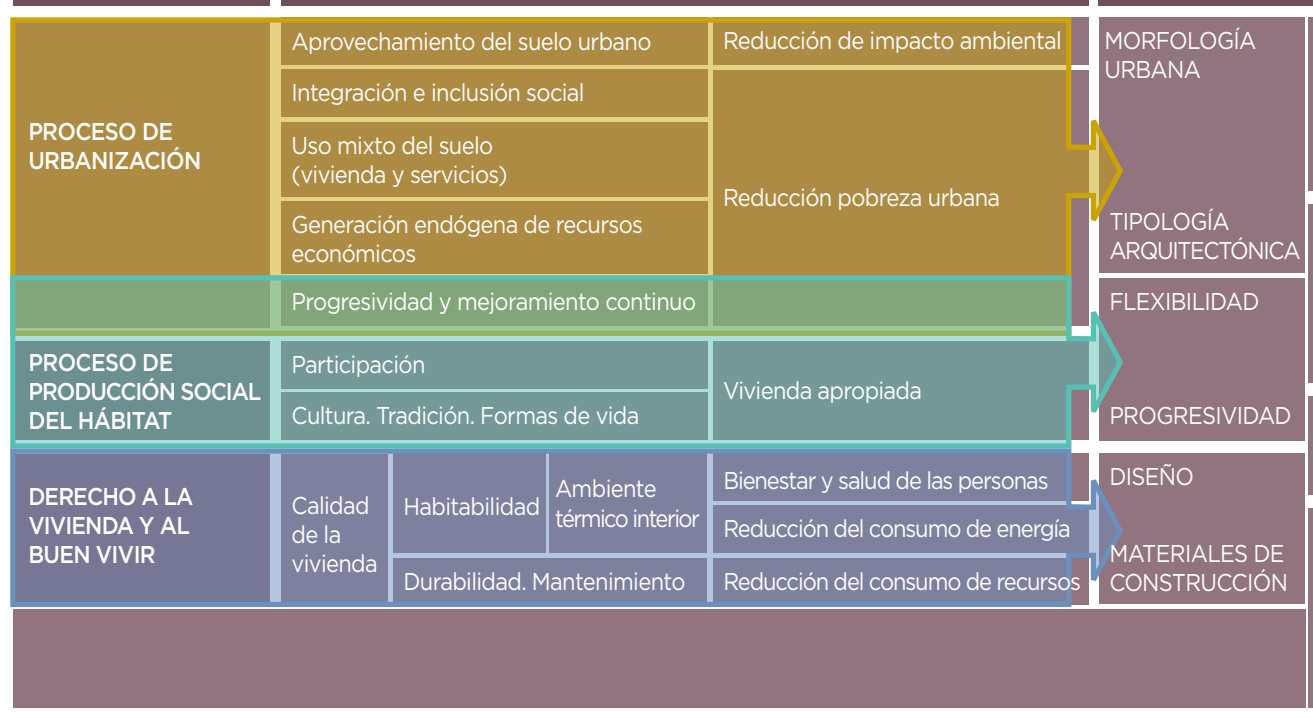

Tabla 1. Variables objeto de estudio que condicionan el ambiente térmico interior en la vivienda (fuente: González y Véliz 2016).
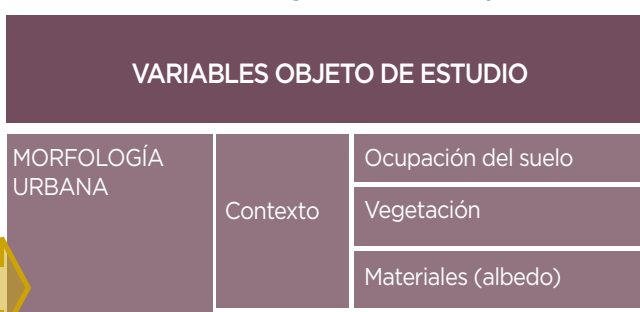

Ocupación del suelo

Vegetación

Materiales (albedo)

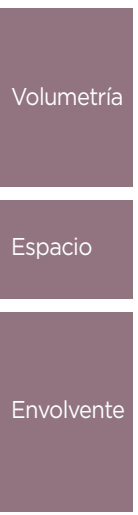

Tipología

$\mathrm{N}^{\circ}$ de plantas

Autosombreado

Dimensiones. Proporciones

Relación con el exterior

Posición. Orientación

Dimensiones. Proporciones

Protección solar

Propiedades físico térmicas

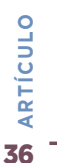


La tipología volumétrica de los edificios y sus formas de agrupación condicionan la ocupación y utilización del suelo, el flujo de viento, las superficies exteriores expuestas o el auto sombreado de la envolvente, en función de la orientación. El espacio interior, sus dimensiones y proporciones, así como su continuidad o compartimentación y su relación con el exterior, influyen en la radiación térmica percibida por las personas y el flujo de aire interior.

La solución volumétrica y espacial de los proyectos de vivienda de interés social se mantiene constante, debido a su carácter repetitivo, aunque suele ser transformada posteriormente por los habitantes. Sin embargo, por tratarse de casos específicos, no es posible definir un solo tipo de vivienda popular autoconstruida. En este sentido, los resultados de García, Bojórquez y Ruiz (2011) son de gran interés, ya que han demostrado que los habitantes de la vivienda popular autoconstruida se adaptan mejor al ambiente térmico interior que los de la vivienda social económica en Mérida, México. Es por ello que en la presente investigación se decidió verificar el resultado comparativo del ambiente térmico interior en la vivienda social y la autoconstruida, a pesar de no constituir una variable identificada como objeto de estudio. Por último, las propiedades físicotérmicas de los elementos y materiales de construcción que conforman la envolvente también tienen gran influencia en el ambiente térmico interior cuando están expuestos a la radiación solar. Esta es la variable más tratada en investigaciones precedentes, que pueden servir como referencia en este estudio exploratorio del ambiente térmico en la vivienda social en el clima cálido y húmedo de Portoviejo, así como también en investigaciones experimentales más detalladas que se encuentran en desarrollo (Romero 2010). Mecott (2007) probó que es posible mejorar el desempeño térmico de los paneles de ferrocemento en la vivienda social en Oaxaca, México. Asimismo, Odeku, Overen y Meyer (2014) han propuesto cómo elevar la eficiencia térmica de viviendas de bajo costo usando pinturas traslúcidas en Sudáfrica. Referencia directa constituye el trabajo desarrollado por Díaz (2012), quien evaluó el desempeño de la cubierta de zinc (acero galvanizado) de la vivienda vernácula en el clima cálido y húmedo de República Dominicana, reconociendo la necesidad de estrategias que contribuyan a mejorar su desempeño térmico, entre las cuales la cubierta doble ventilada aparece como preferible.

También se ha demostrado ampliamente en investigaciones precedentes que las cámaras de aire contribuyen a reducir la transferencia térmica hacia el interior, principalmente si están ventiladas. En un estudio experimental, Lee, Park y Yeo (2009) demostraron que la reducción del flujo térmico aumenta con el incremento del ángulo de inclinación de la cubierta, el espesor de la cámara de aire y la temperatura superficial.

En otra investigación, Kabre (2010) propuso un nuevo índice para evaluar el desempeño térmico de las cubiertas de viviendas en clima tropical húmedo (TPI), considerando la temperatura de la superficie interior de la cubierta y asignando un valor equivalente a cero para el zinc (peor condición) y uno para aquel que satisfaga las necesidades de

\section{CONTEXTO: PORTOVIEJO}

Portoviejo. Portoviejo está situada en una latitud de $1^{\circ}$ Sur, con una altitud de 53 m sobre el nivel medio del mar. La humedad relativa es alta todo el año, con medias mensuales que oscilan entre $67 \%$ en noviembre y $79,5 \%$ en junio. Sin embargo, resulta significativa la amplia oscilación de los valores diarios, cercanos a $100 \%$ en la noche, y entre $40 \%$ y $60 \%$ durante el día. Se identifican dos estaciones, una lluviosa conocida como 'invierno' de enero a abril, en la que la lluvia puede alcanzar los $132 \mathrm{~mm}$, y otra seca de mayo a diciembre, cuando las precipitaciones se reducen a $6 \mathrm{~mm}$. La nubosidad es muy alta durante todo el año (entre 6 y 7 octavos), excepto en abril y septiembre, como resultado de lo cual el indice de horas de sol promedio anual es de 3,5 horas. Los vientos predominantes provienen del Noreste durante casi todo el año, y del Sudeste en mayo, junio, agosto y octubre, con bajas velocidades medias que oscilan entre $1,4 \mathrm{~m} / \mathrm{s}$ en febrero y agosto y $1,9 \mathrm{~m} / \mathrm{s}$ en mayo.

Al visibilizar la combinación de valores máximos y mínimos de temperatura con los mínimos y máximos de humedad relativa para cada mes en la Carta Bioclimática de Givoni (1998) (figura 1), se puede apreciar que las condiciones diurnas están fuera de la zona de confort, en el área correspondiente a la estrategia de enfriamiento mediante ventilación natural o mecánica, lo cual es difícil en Portoviejo debido a las reducidas velocidades del viento predominante. Algunos puntos se ubican en la zona correspondiente al posible enfriamiento evaporativo, gracias a los bajos valores de humedad relativa al mediodía.

Por otro lado, la combinación de valores medios de la temperatura mínima y la humedad relativa máxima representativos del período nocturno también se encuentran fuera de la zona de confort, pero en este caso no por la temperatura -que es relativamente baja (entre $19^{\circ} \mathrm{C}$ y $22^{\circ} \mathrm{C}$ )-, sino debido a la alta humedad relativa (superior a 90\%). No obstante, se ha verificado que los valores reales de la temperatura del aire en las áreas urbanas estudiadas son superiores a aquellos registrados simultáneamente en la estación meteorológica.

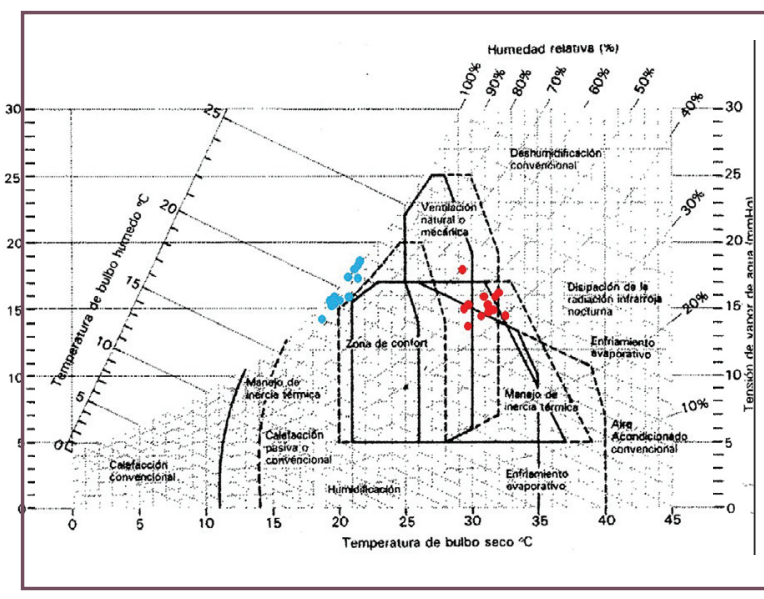

Figura 1. Estrategias de diseño recomendables según Givon (1998) para las condiciones climáticas de Portoviejo (fuente: Elaboración propia). 
Según la metodología de Mahoney (Chidambareswaran 2005), para las condiciones de Portoviejo -cálidas durante el día y muy húmedas en la noche- las estrategias de diseño recomendables pueden resumirse como máxima protección solar y permeabilidad al viento, con cubiertas ligeras y aisladas de bajo coeficiente global de transferencia térmica

y paredes pesadas.

\section{Vivienda social promovida por el Ministerio de Urbanismo y Vivienda (MIDUVI).}

desarrollados por el MIDUVI se basan repetitivos. El primero consistió en una vivienda de $42 \mathrm{~m}^{2}$, de planta cuadrada, en dos crujías, con cubierta de acero galvanizado, inclinada a dos aguas sobre viguetas apoyadas en estructura de vigas y columnas de hormigón armado, y paredes exteriores de bloques de mortero o ladrillo cerámico, con ventanas cuadradas de vidrio al frente y al fondo, todo lo cual conforma la envolvente. Las condiciones térmicas interiores estaban, adicionalmente, afectadas por la ausencia de aleros y portal. Este modelo descrito fue posteriormente sustituido por otro que intentaba mejorar la imagen de la vivienda, transformando la fachada principal, sin resolver los problemas ambientales, ya que mantenía la cubierta ligera de alta transmitancia y las ventanas de vidrio expuestas al sol (figura 2). A diferencia del modelo inicial, los calados para favorecer la ventilación y el enfriamiento estructural se sustituyeron por bloques de vidrio que solo beneficiaban la iluminación natural. en el empleo de dos tipos de proyectos

MATERIALES Y MÉTODOS. Selección de casos de estudio. del ambiente térmico interior en la vivienda de interés social promovida por el MIDUVI (20132014), para seleccionar los casos de estudio se identificaron las áreas urbanas donde se han construido más viviendas de ese prototipo durante los últimos años: Colón, El Florón, Las Orquídeas, Ciudadela San Jorge (Picoazá) y María Asunción (Picoazá). En cada una de ellas se seleccionó una muestra intencional que incluyó todas las viviendas de este tipo, así como otras autoconstruidas por la población. Estas últimas fueron seleccionadas de forma aleatoria.

La muestra quedó integrada por 92

viviendas: 74 correspondientes a proyectos de MIDUVI y 18 viviendas populares autoconstruidas con diferentes materiales. Todas fueron caracterizadas en función de las variables objeto de estudio, con la finalidad de comparar los resultados.

Contexto urbano. Se trata de urbanizaciones periféricas con pobre infraestructura, habitadas por población de escasos recursos, con características comunes en cuanto a la morfología urbana dispersa, de muy baja densidad, con ausencia de calles conformadas y muy pocas áreas pavimentadas, por lo cual la influencia del contexto en el ambiente térmico interior no es considerable. Es por ello que en la caracterización de la muestra con respecto a la influencia del contexto en la temperatura interior, solo se consideró la presencia cercana de edificios o árboles (aquellos ubicados a menos de $10 \mathrm{~m}$ de la vivienda objeto de estudio).

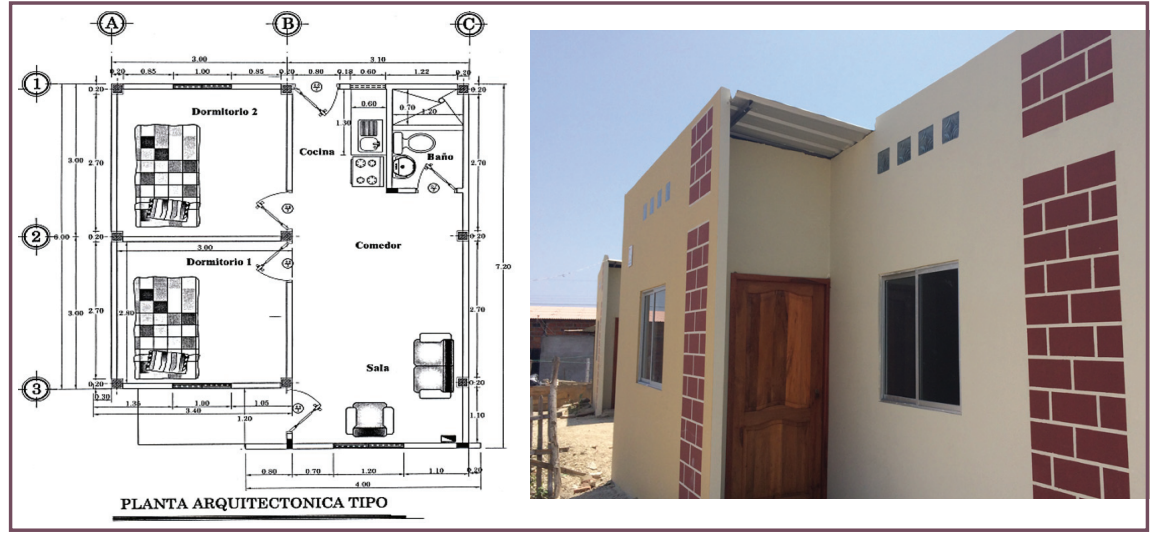

Solución volumétrica. Las tipologías volumétricas consideradas han sido: aislada, pareada, en hilera, y en una y dos plantas, teniendo en cuenta las cuatro orientaciones principales. Predominan en la muestra viviendas unifamiliares aisladas de una planta, directamente apoyadas en el terreno, que responden al proyecto repetitivo de MIDUVI, excepto en María Asunción de Picoazá, donde se presentan viviendas en hilera de planta baja libre, que constituyen un proyecto atípico (imagen 1). La muestra incluye viviendas con fachada hacia las cuatro orientaciones principales ( $N, S$, E y O).

\section{Envolvente. Materiales de}

construcción. Las viviendas estudiadas presentan cuatro tipos de cubierta: pesada de hormigón armado; ligera, con láminas de acero galvanizado; duratecho2; y de fibras vegetales ('cady') sobre estructura de viguetas.

Las paredes de bloques y las de ladrillo se encuentran en igual proporción en la muestra estudiada, que incluye algunas viviendas populares con paredes de bambú, para comparar los resultados obtenidos en las mediciones de la temperatura del

\section{aire interior}

\section{Experimentación. Se realizaron}

mediciones de temperatura y humedad relativa del aire en 92 viviendas, mediante el empleo de un "Pocket Air Flow Tracker (Kestrel 4200)" situado en el estar, a $1,50 \mathrm{~m}$ sobre el nivel de piso terminado, programado para realizar registros horarios (imagen 2). De forma simultánea, se hicieron mediciones en un punto exterior de referencia ubicado a menos de 10 $m$ de la vivienda estudiada, usando el mismo instrumento situado en una cabina meteorológica bajo un árbol a 2 m sobre el terreno (imagen 3). También se verificó la percepción de los habitantes del ambiente térmico interior mediante entrevistas. Para comparar los resultados de mediciones no simultáneas se usó la diferencia de temperatura $(\Delta t)$ entre el interior y el exterior de referencia. Las temperaturas exteriores fueron también comparadas con los registros simultáneos en la estación meteorológica, para así evaluar la posible influencia del contexto urbano en el microclima local y verificar el efecto de la isla de calor en cada sector 


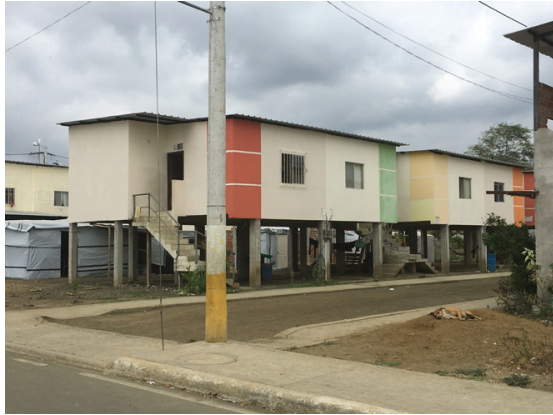

Imagen 1. Vivienda en hilera en María Asunción de Picoazá (fuente: Los autores).

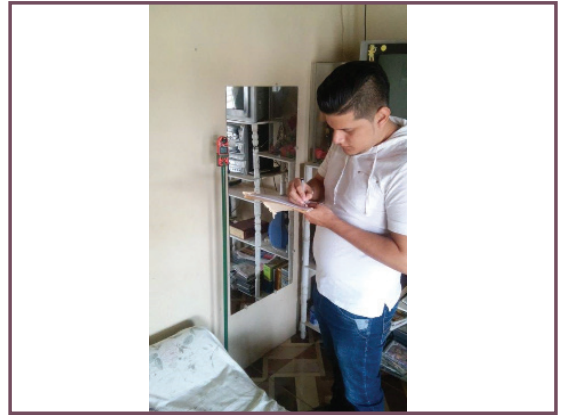

Imagen 2. Equipo de medición en el interior de una vivienda en Colón (fuente: Los autores).

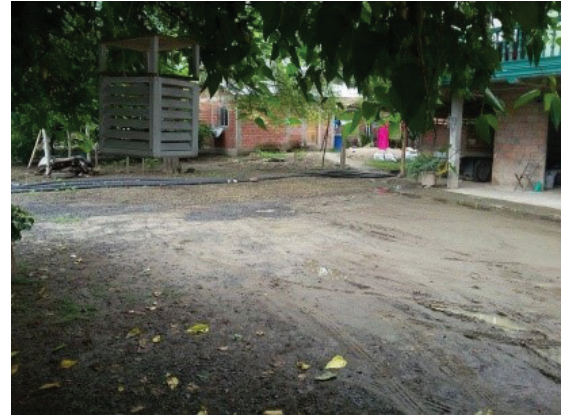

Imagen 3. Equipo de medición en un punto de referencia exterior en Colón (fuente: Los autores).

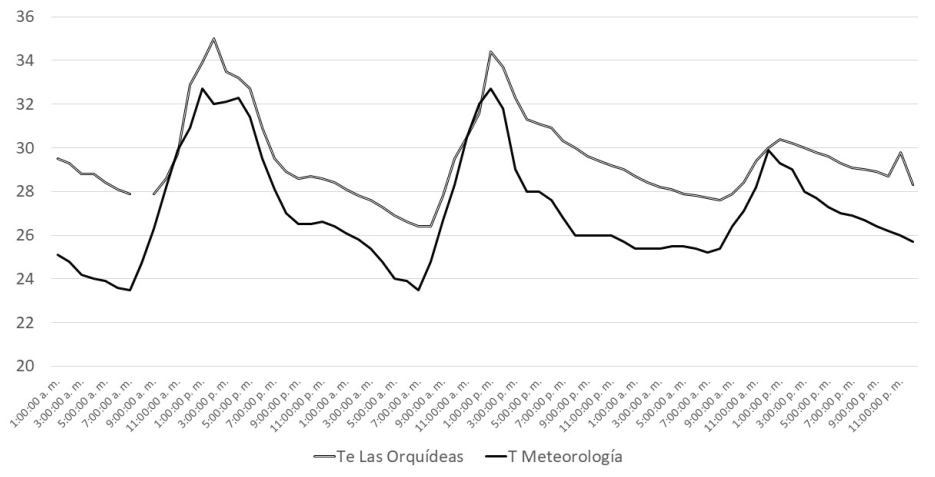

Figura 3. Temperatura del aire exterior en Las Orquídeas con respecto a la estación meteorológica (fuente: Elaboración propia).

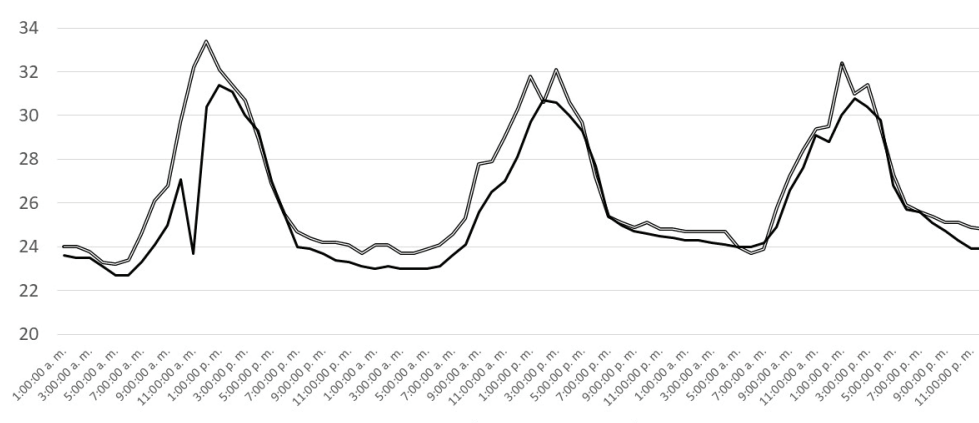

一Te Picoazá -T Meteorología

Figura 4. Temperatura del aire exterior en San Jorge de Picoazá con respecto a la estación meteorológica (fuente: Elaboración propia).

\section{RESULTADOS Y DISCUSIÓN.}

Isla de calor urbana. Según lo esperado,

la temperatura del aire en los puntos exteriores de referencia fue superior a la medida simultáneamente en la estación meteorológica. Las mayores temperaturas se registraron en Las Orquídeas, situada en la zona urbana más compacta y consolidada de las estudiadas (figura 3), con diferencias de hasta $4^{\circ} \mathrm{C}$ en la noche.

La menor diferencia de temperatura entre la referencia exterior y la estación meteorológica se encontró en las dos urbanizaciones de Picoazá, localizadas hacia la periferia, con mayores valores de $\Delta$ t durante la mañana y muy pequeñas diferencias (menos de $1^{\circ} \mathrm{C}$ ) en la noche (figura 4).

Al visibilizar, en la Carta Bioclimática de Givoni, los valores máximos y mínimos de temperaturas y humedades exteriores, se observó un desplazamiento hacia valores mayores de humedad relativa en la noche y temperaturas superiores con menor humedad al mediodía (figura 5). Por lo tanto, las condiciones micro climáticas nocturnas están mayormente en la zona de confort o donde estas pueden obtenerse mediante ventilación natural o mecánica, mientras que es mucho más difícil alcanzar el confort durante el día en las zonas urbanas, incluso usando ventilación.

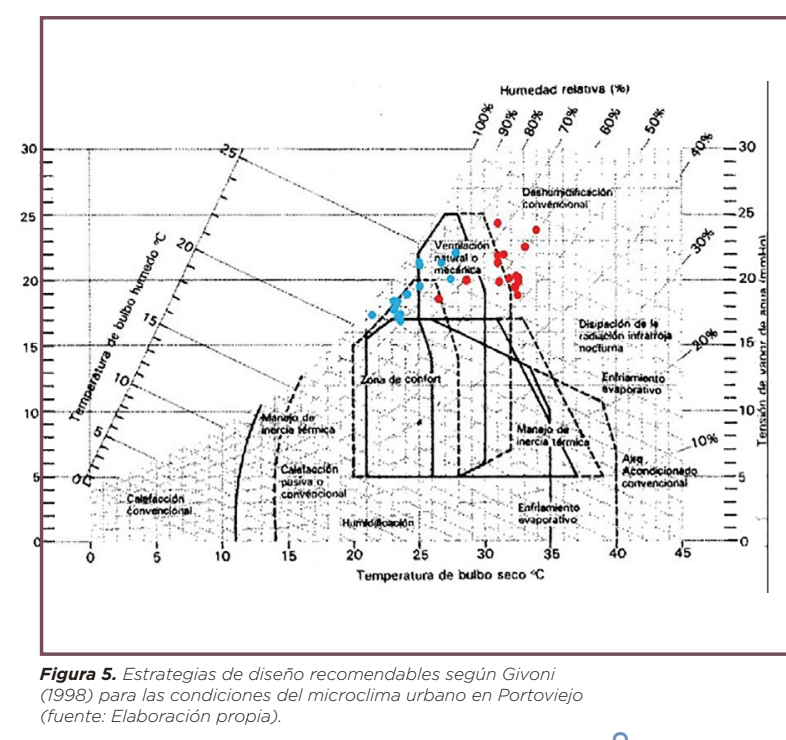


6

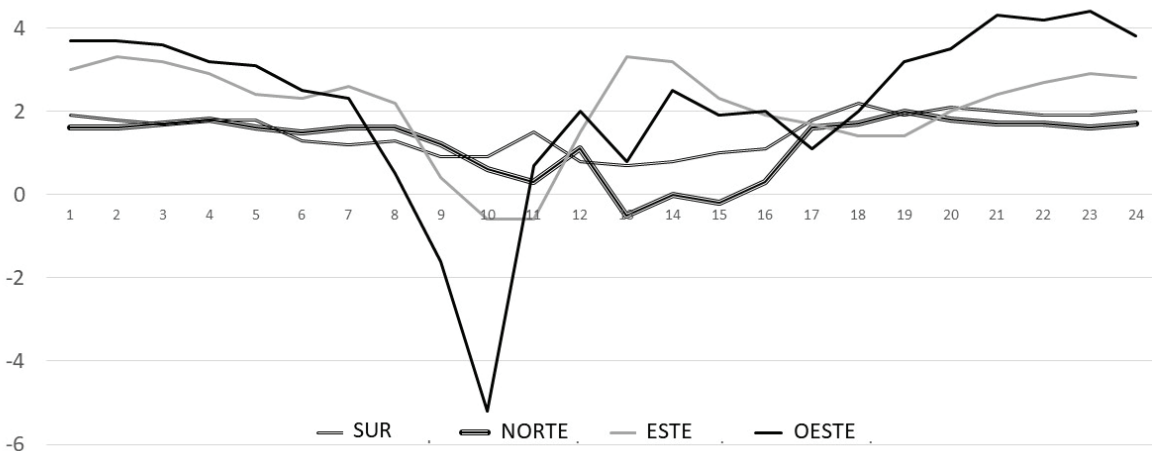

Figura 6. Diferencia entre la temperatura del aire interior y exterior en vivienda del MIDUVI aislada de una planta con techo de zinc y paredes de ladrillo en diferentes orientaciones (fuente: Elaboración propia).

INFLUENCIA DEL MATERIAL DE CONSTRUCCIÓN DE LA PARED EN LA TEMPERATURA INTERIOR

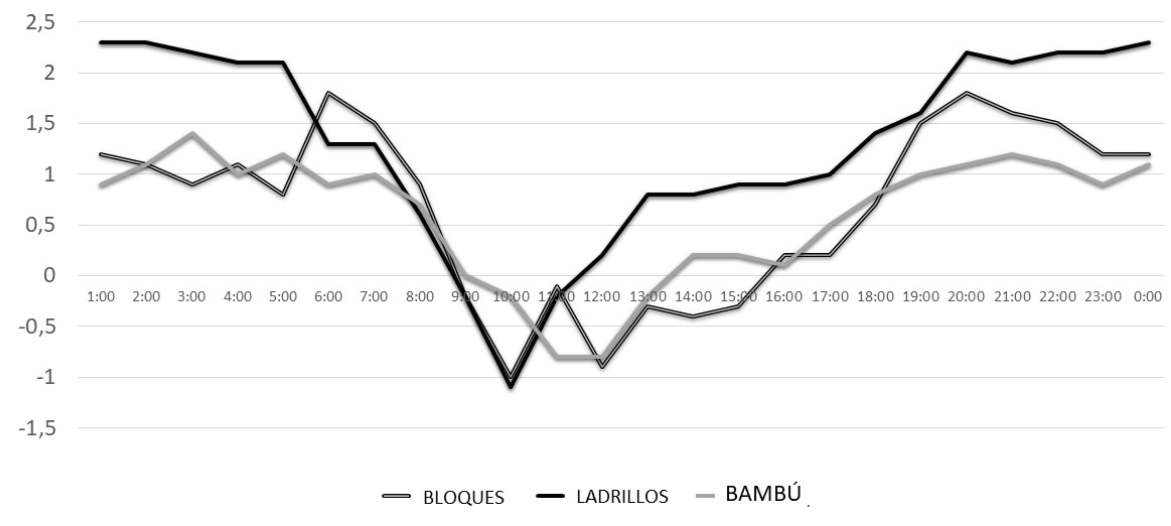

Figura 7. Diferencia entre la temperatura del aire interior y exterior en viviendas aisladas de una planta con fachada orientada al Norte, techo de zinc y paredes hechas de bambú, bloques de mortero y ladrillos (fuente: Elaboración propia).

INFLUENCIA DEL MATERIAL DE CONSTRUCCIÓN DE LA CUBIERTA EN LA TEMPERATURA INTERIOR

$$
6
$$

$$
5
$$$$
5
$$$$
4
$$$$
3
$$

2

1

0

$-1$

$-2$

$$
\text { - DURATECHO - ZINC }
$$

$$
\text { - HORMIGÓN }
$$

它
Contexto y tipología. Es significativo que viviendas que responden a proyectos del MIDUVI con diferente tipología (aislada y pareada) arrojan similares condiciones térmicas que no son las más favorables en la noche y, al mismo tiempo, son las peores durante el día, con una temperatura interior de $4^{\circ} \mathrm{C}$ superior a la exterior a las $3: 00 \mathrm{pm}$, aun con vegetación cercana. Sin embargo, otras viviendas populares autoconstruidas también presentan similar desempeño, aunque más favorable, con una temperatura interior siempre mayor que la exterior, pero en menos de $1^{\circ} \mathrm{C}$. La vivienda en hilera de Picoazá también es satisfactoria, teniendo en cuenta que la temperatura interior es mayor que la exterior, pero en menos de $1^{\circ} \mathrm{C}$, e incluso inferior a las 11:00 am. Sin embargo, esta tipología es menos aceptada por la población que prefiere la vivienda aislada.

Orientación. Para verificar la influencia de la orientación en la temperatura del aire interior se compararon los resultados de las mediciones realizadas en viviendas del MIDUVI aisladas, de una planta con techo de zinc y paredes de ladrillo, en diferentes orientaciones. Según lo esperado, no hay grandes diferencias entre las orientaciones Norte y Sur, como consecuencia de la baja latitud, y tampoco significativas con respecto al Este (figura 6). Sin embargo, la orientación Oeste genera temperaturas interiores inferiores durante la mañana, pero superiores en la noche. Esto demuestra que la prioridad respecto al Sur o el Norte no es significativa aquí, pero la orientación Oeste continúa siendo la peor condición, que debe evitarse para los espacios habitables de función principal en los climas cálidos, especialmente aquellos que, como los dormitorios, se utilizan durante la noche. 
Material de la pared. Para evaluar la influencia del material de la pared en la temperatura del aire interior, se compararon tres casos de estudio que constituyen soluciones unifamiliares aisladas, cercanas a otra edificación, con fachada orientada al Norte y cubierta de 'duratecho', pero con diferentes materiales de pared: bloques de mortero, ladrillos y bambú. El mejor resultado se obtiene en la vivienda con paredes de bambú, con una temperatura interior superior a la exterior en solo $1^{\circ} \mathrm{C}$. El peor desempeño corresponde a la vivienda de paredes de ladrillo, con temperatura interior superior a la exterior en $1^{\circ} \mathrm{C}$ durante toda la tarde, y más de $2^{\circ} \mathrm{C}$ en la noche (figura 7). Sin embargo, las fibras naturales (como las paredes de bambú) ya no se utilizan en la vivienda, ya que son escasas y las personas las asocian con pobreza y

\section{subdesarrollo.}

Material de cubierta. Se tomaron tres de las viviendas estudiadas para evaluar la influencia del material de cubierta en la temperatura interior. Estas son de un piso, con la fachada orientada al Norte y cubierta de 'duratecho', zinc u hormigón. El mejor desempeño se observa en aquellas con 'duratecho', donde se mantiene una temperatura interior mayor que la exterior en menos de $1^{\circ} \mathrm{C}, \mathrm{y}$ aun menor que la exterior también en $1^{\circ} \mathrm{C}$ a las 11:00 am. El hormigón armado es la peor solución, con valores de la temperatura interior mayores que la exterior en hasta $4^{\circ} \mathrm{C}$. El techo de zinc presenta un comportamiento intermedio, con la temperatura interior hasta $2^{\circ} \mathrm{C}$ por encima de la exterior en la noche y $1^{\circ} \mathrm{C}$ durante el día (figura 8 ).

Como puede apreciarse, a pesar de que la solución de cubierta de uso más extendido (zinc) no presenta el mejor desempeño térmico, es mucho mejor que el hormigón armado, que es la solución ideal, durable, 'moderna' y 'desarrollada' a la cual muchas personas aspiran y que usan tan pronto como las condiciones económicas se lo permiten.

CONCLUSIONES. El estudio exploratorio realizado permite verificar el efecto de la isla de calor urbana. No obstante, no se aprecia la influencia del contexto urbano en la temperatura del aire interior, por tratarse en todos los casos de urbanizaciones periféricas dispersas con bajo albedo. Al mismo tiempo, tampoco hay diferencias notables según la tipología de vivienda aislada o pareada. Sin embargo, la vivienda en hilera presenta condiciones ambientales interiores más favorables. También es posible apreciar que la vivienda popular autoconstruida presenta mejor ambiente térmico interior que la desarrollada por el MIDUVI. Con respecto a la orientación, nuevamente las diferencias no son significativas, pero la orientación Oeste es menos favorable durante la tarde y noche.
Cuando las soluciones tipológicas de las viviendas y los contextos son similares (como en el presente estudio), los materiales de construcción constituyen la variable que más influye en la temperatura del aire interior. A pesar de que según Mahoney (Chidambareswaran 2005), los paredes pesadas con cierta inercia térmica podrían ser recomendables para las condiciones climáticas de Portoviejo, las temperaturas interiores menores se obtienen en viviendas con paredes de bambú y las mayores con ladrillo, cuya inercia térmica es superior a la de los bloques de mortero. Estos resultados también coinciden con la percepción térmica manifestada por las personas. Por otro lado, las mayores temperaturas interiores se registran en viviendas con cubiertas de hormigón armado, fundamentalmente en la noche, lo cual concuerda con la percepción de los habitantes. La población entrevistada considera de forma casi unánime que sus viviendas son calurosas o muy calurosas, sobre todo durante el día, y aún más en la tarde, con excepción de los habitantes de las viviendas con paredes de bambú, quienes manifestaron que estas eran relativamente frescas durante el día. Por el contrario, los que viven en viviendas con cubierta de hormigón armado, dicen que estas son muy calurosas, incluso en la noche. $\mathbf{\Delta u}$

\section{REFERENCIAS}

Alonso, N., Sarachu, G. y Vallés, R., 2012. "Dos modelos y sus resultados. Producción habitacional por empresas "llave en mano" y por cooperativas." Revista Vivienda Popular, 22, 71-79, Chidambareswaran, S., 2005. Mahoney Tables p/us a tool for sketch design recommendations for a building. California: University of Southern California. Disponible en: http://digitallibrary.usc.edu/cdm/ref/ collection/p15799coll16/id/45199

Cravino, C., 2012. "Habitar nuevos barrios de interés social en el área metropolitana de Buenos Aires: el espacio construido por el Estado y vivido por los vecinos". En FLACSO, Dimensiones del hábitat popular Jatinoamericano. Quito: FLACSO Ecuador, 101-120.

Díaz, 0. 2012. La cubierta metálica en el clima cálido húmedo: Análisis de/ comportamiento térmico del techo de zinc de la vivienda vernácula dominicana. Barcelona: Universidad Politécnica de Cataluña. Disponible en: https://upcommons.upc.edu/bitstream/handle/2099.1/16062/DiazOsttuhen_TFM.pdf.

Espinosa, C. y Cortés, A., 2015. "Confort higrotérmico en vivienda social y la percepción del habitante." Revista INVI. 85, 227-242

García, C., Bojórquez, G. y Ruiz, P., 2011. "Sensación térmica percibida en vivienda económica y autoproducida, en periodo cálido, para clima cálido húmedo." Ambiente Construido, 11 (4): 99-111.

Givoni, B., 1998. Climate Consideration in Building and Urban Design. Nueva York: Van Nostrand Reinhold. González, D. y Sánchez, O., 2016. "Towards a more resilient urban morphology in Havana." PLEA 2016: 32nd International Conference on Passive and Low Energy Architecture. University of Pomona.

González, D. y Véliz, J., 2016. "Resiliencia urbana y ambiente térmico en la vivienda." Arquitectura y Urbanismo. 37 (2): $63-73$.

González, D., 1997. Economía y calidad en la vivienda. Un enfoque cubano. La Habana: Editorial Cientifico Técnica.

Kabre, Ch., 2010. "A new thermal performance index for dwelling roofs in the warm humid tropics." Building and Environment, 45: 727-738.
Lee, S., Park, S. y Yeo, M., 2009. "An experimental study on airflow in the cavity of a ventilated roof". Building and Environment, 44, 1431-1439.

Mecott, S., 2007. Vivienda bioclimática con paneles modulares de ferro cemento y materiales ais/antes alternativos para la ciudad de Oaxaca. (Tesis para optar al grado de Magíster en Ciencias en Conservación y Aprovechamiento de Recursos Naturales, Instituto Politécnico Nacional, Centro Interdisciplinario de Investigación para el Desarrollo Integral Regional, Unidad Oaxaca, México). Disponible en: https://tesis.ipn.mx/ jspui/handle/123456789/155

Ministerio de Desarrollo Urbano y Vivienda (MIDUVI), 2013. DAP Aclaración vigencia prioridad "Programa Nacional de Vivienda Social", MIDUVI. Disponible en: www.habitatyvivienda.gob.ec/wp-content/uploads/ downloads/2015/06/PROYECTO-PROGRAMA-NACIONAL-DE-VIVIENDA-SOCIAL.pdf Odeku, K., Overen, K. y Meyer, E., 2014. "Thermal Efficiency for Low Cost Houses using Translucent WaterBased Acrylic Paint." Mediterranean Journal of Social Sciences, 5 (20): 2961-2966. Disponible en: http://www. mcser.org/journal/index.php/mjss/article/view/4428/4329

Pérez, A., 2013. Bases para el diseño de la vivienda de interés social: según las necesidades y expectativas de los usuarios. Bogotá: Universidad de La Salle.

Ramos, H., 2012. Metodología para diagnosticar la habitabilidad en la vivienda social, Bogotá: Universidad de La Salle.

Romero, R., 2010. "Confort térmico y ahorro de energía en la a vivienda económica en México: Regiones clima cálido, seco y húmedo." Primer Encuentro Académico CONAVI CONACID. México. Disponible en: http://20062012.conacyt.gob.mx/fonfos/FondosSectoriales

Staines, E., 2013. Habitabilidad y eficiencia energética en conjuntos habitacionales de interés social. Ciudad Juárez: Universidad Autónoma de Ciudad Juárez. Disponible en: https:repositorio.uchile.cl/bitstream/ handle/2250/118201/habitabilidad-tapia.pdf.

Vargas, G., Walsh, C. y Barros, M., 2016. "Evaluación cuali-cuantitativa e aislaciones térmicas alternativas para viviendas. El caso de la agricultura familiar." Revista INVI, 86, 89-117. 